\title{
Dual Lens Electron Holography for High Spatial Resolution Junction and Strain Mapping of Semiconductor Devices
}

\author{
Y.Y. Wang and J. Bruley \\ IBM, Zip 40E, Hudson Valley Research Park, 2070 Route 52, Hopewell Jct., NY 12533, USA
}

Electron holography has been shown to be an affective tool to measure junction profile and strain map at nanometer scale.[1,2] However, in a convention transmission electron microscope setting, where the magnification of the objective lens is usually fixed and the magnification is controlled by the projection lenses, the field of view (FOV) of electron holography is limited. In order to make electron holography more flexible, adjustable FOV and spatial resolution relative to the object is desirable. A method of using two objective lenses (dual lens) to provide a continuously varying magnification of objective lenses at a fixed intermediate imaging plane was developed.[3-6] The dual lens operation allows electron holography to be performed from low to high magnifications and provides the FOV and fringe spacing necessary for two-dimensional (2D) junction mapping and strain profiling of semiconductor devices.

Fig. 1 shows the principle of dual lens operation, where a magnified virtual image is formed by the first lens and a real image is projected by the second lens. Adjusting the first lens in combining with second lens allows magnification to be changed without changing the initial object position and final imaging plane. Fig. 2 shows the results of fringe spacing and fringe width relative to the object in relationship with the excitation of the first lens. With increasing of objective lens current, the fringe spacing continuously decreases from $4 \mathrm{~nm}$ to $0.3 \mathrm{~nm}$ and field of view from $700 \mathrm{~nm}$ to $100 \mathrm{~nm}(7 \mathrm{x}$ change). This allows us to obtain the best imaging condition for various device dimensions.

Fig. 3 shows four examples of junction and strain maps for various devices. In the semiconductor industry, low dose implant (also known as extension implant) is used near the gate region to control the gate leakage and high dose implant in source/drain region is used for better conduction. Fig.3a is the junction profile for pFET with bulk Si technology. In the map, the sharp junction in the extension region can be observed clearly under the gate. In the source/drain region, the bottom of the p$\mathrm{n}$ junction is much more diffused than the one under the gate because of high implant dose. Fig. $3 \mathrm{~b}$ is the nFET junction profile with a shallow junction in the extension. In that profile, we observed that $\mathrm{CoSi}_{2}$ is too close to the point where extension region and source/drain region cross each other. This shows that in order to make shallow junction technology work, raised source/drain is needed to move silicide away from the junction.

In semiconductor industry, to further reduce the device size and increase device performance, strain technology is introduced. For pFET, compressive strain is needed for device enhancement and for nFET, tensile strain is needed to improve device performance. Adding strain into the device increases the process step, development cost, and cycle time. Strain profiling under the gate at high spatial resolution is extremely useful to reduce development cost and cycle time. Fig.3c shows high spatial resolution strain map on bulk Si pFET with compressive strain along $<220>$ under the channel. Because of the sigma shaped SiGe, the strain is measured under the channel as high as $-1.7 \%$ (blue color). Fig.3d shows high spatial resolution strain map along $<220>$ direction for nFET devices with stress memorization technique (SMT) process. Initially, the mechanism of stress memorization is not well understood. With high spatial resolution map, it shows that stress is memorized in the dislocation (shown bright yellow/blue dumbbell) and tensile strain under the channel is due to the dislocation on both sides of the gate. The highest strain is measured as $1.1 \%$ under the gate (red color).[7] 
In conclusion, dual lens electron holography allows us to obtain high spatial resolution junction and strain map of semiconductor device. We would like to acknowledge the support of IBM managers for the development of the dual lens electron holography technique.

\section{References:}

1).M. Hytch et al., Nature 453 (2008) 1086

2).W.D. Rau et al., Physical Review Letter 82 (1999) 2614

3).Y.Y. Wang et al., Ultramicroscopy 101 (2004) 63

4).Y.Y. Wang et al., US patent: US 7,015,469 B2 (2006)

5).Y.Y. Wang et al., JEOL News 47 (2012) 9

6).Y.Y. Wang et al., Ultramicroscopy 124 (2013) 117

7).Y.Y. Wang et al., Applied Physics Letter 103 (2013) 052104.

Fig.1: Optical ray diagram of dual lens operation; Fig.2: fringe spacing and fringe width relative to the object vs objective lens current with a fixed biprime voltage; Fig.3: Junction and strain maps obtained by dual-lens electron holography.

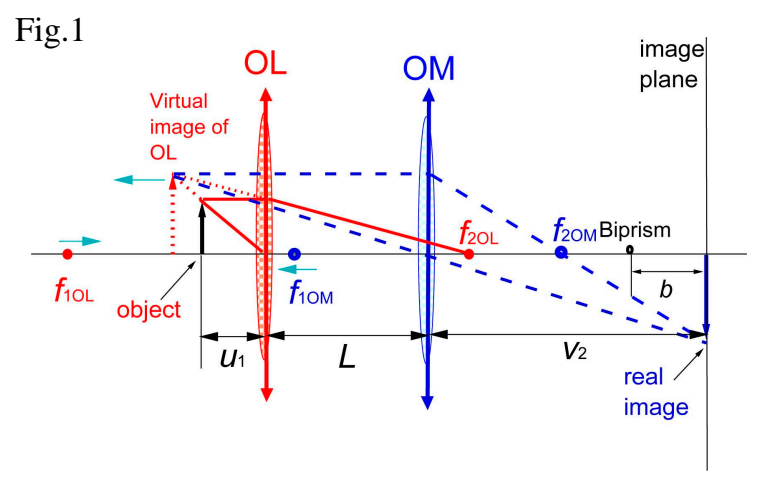

Fig.2

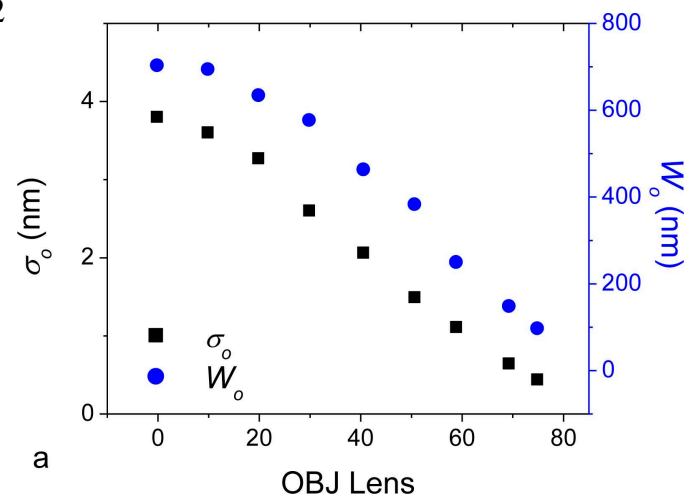

Fig.3

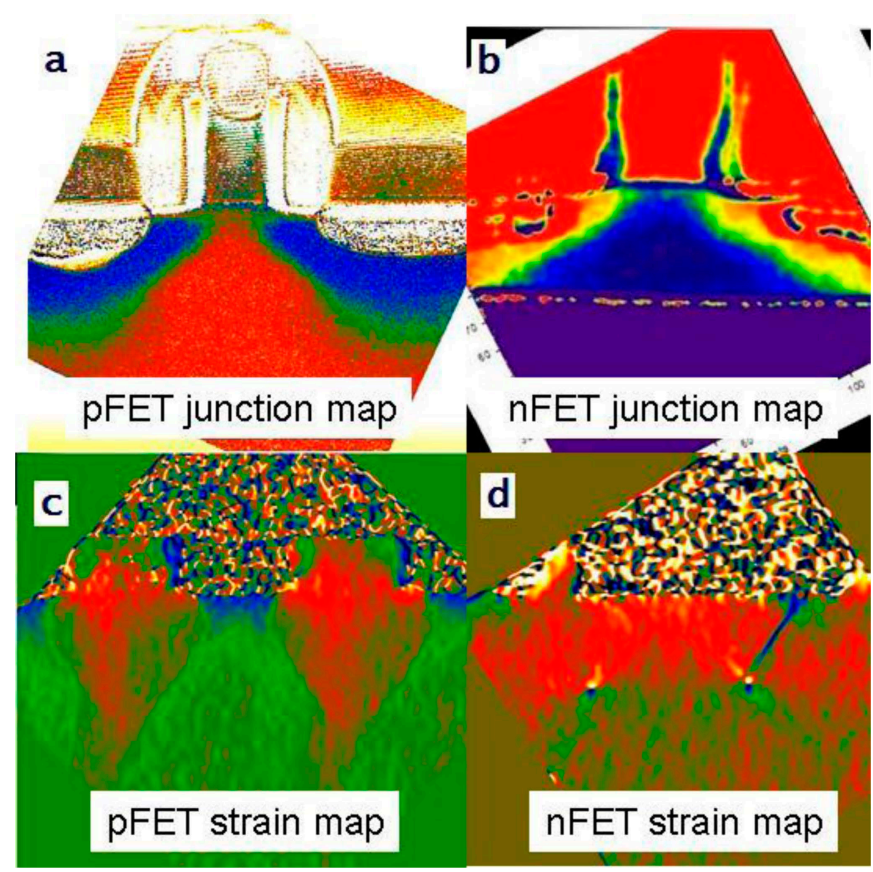

